\title{
Searching for Compact Objects in Supernova Remnants: Initial Results
}

\author{
D. L. Kaplan, S. R. Kulkarni \\ Department of Astronomy, 105-24 California Institute of Technology, \\ Pasadena, CA 91125, USA \\ D. A. Frail
}

National Radio Astronomy Observatory, P.O. Box O, Socorro, NM 87801, USA

B. M. Gaensler, P. O. Slane

Harvard-Smithsonian Center for Astrophysics, 60 Garden Street, MS-6, Cambridge, MA 02138, USA

E. V. Gotthelf

Columbia Astrophysics Laboratory, Columbia University, 550 West 120th Street, New York, NY 10027, USA

\begin{abstract}
Most astronomers now accept that stars more massive than about $9 \mathrm{M}_{\odot}$ explode as supernovae and leave stellar remnants, either neutron stars or black holes. However, less than half of the SNRs within $5 \mathrm{kpc}$ have identified central sources. Here, we discuss a systematic effort to search for compact central sources in the remaining 23 SNRs of this distance-limited sample. As the first part of this survey, we are able to state with some confidence that there are no associated central sources down to a level of one tenth of that of the Cas A central source, $L_{X} \lesssim 10^{31} \mathrm{ergs} \mathrm{s}^{-1}$, in four SNRs (G093.3+6.9, G315.4-2.3, G084.2+0.8, and G127.1+0.5). We compare our limits with cooling curves for neutron stars and find that any putative neutron stars in these SNRs must be cooling faster than expected for traditional $1.35 \mathrm{M}_{\odot}$ neutron stars.
\end{abstract}

\section{Introduction}

Understanding the deaths of massive stars is one of the frontiers of modern astrophysics. The discovery of pulsars in the Vela SNR and the Crab Nebula made concrete the suggestion that core collapse results in neutron stars (Baade \& Zwicky 1934), some of which manifest themselves as radio pulsars. However, recent developments have severely revised our picture of young neutron stars. Most importantly, astronomers have come to accept of tremendous diversity in the natal properties of young neutron stars. Anomalous X-ray pulsars (AXPs; Mereghetti et al. 2002), soft $\gamma$-ray repeaters (SGRs; Hurley 2000), nearby ther- 
mal and radio quiet neutron stars (Pavlov et al. 2002), long period radio pulsars with high inferred magnetic fields (HBPSR; Camilo et al. 2000; Gotthelf et al. 2000) are now routinely found in the astronomical literature. These new classes of neutron stars have primarily come from high energy observations.

\section{Survey Design}

Three years ago we began a program to observationally investigate the stellar remnants in a volume-limited census of Galactic supernova remnants. The basis of our effort is that observationally, all central sources in SNRs known to date, regardless of the band of their initial identification ( $\gamma$-ray, X-ray, or radio) appear to possess detectable X-ray emission. Theoretically, we expect thermal X-ray emission from young neutron stars. Thus, on both counts the search for central sources in young remnants is very well motivated.

To this end, we have identified a sample of SNRs within $5 \mathrm{kpc}$ of the Sun. Most of these SNRs are expected to contain central neutron stars: $\lesssim 20 \%$ are expected to result from Type Ia $\mathrm{SNe}$ and thus not contain a central compact source, while $\sim 20 \%$ are expected to host a central black hole that may not be easily identified as such. And in fact about half of these SNRs have known neutron stars at their centers. We are then left with the SNRs that have no obvious indication of central sources: the hollow SNRs. We successfully proposed for a "large" Chandra effort in AO-3 to image the nine smallest of these SNRs. Here, we report the first analysis of four SNRs for which the followup is now complete: SNRs G093.3+6.9, G315.4-2.3, G084.2+0.8, and G127.1+0.5. For more details, see Kaplan et al. (2004).

After identifying X-ray sources with Chandra, the question is then to determine which, if any, are the compact remnants of the SNRs. Because of their small X-ray count-rates, weeding out interlopers requires multi-wavelength observations. Isolated neutron stars have high X-ray to optical flux ratios (e.g., Hulleman, van Kerkwijk \& Kulkarni 2000). Interloper sources, on the other hand, typically have much brighter optical/IR counterparts (Hertz \& Grindlay 1988; Grindlay et al. 2003). In the Galactic plane, the majority of sources are either nearby bright stars or active late-type stars. The extragalactic sources are usually AGN or star-forming galaxies, although some nearby spiral galaxies are also detectable (Barger et al. 2003). Deep optical/IR imaging is therefore an efficient way to identify background sources, and we follow our X-ray observations with successively deeper optical and IR observations, identifying progressively fainter counterparts as we go.

\section{Initial Results}

With deep optical and infrared imaging we were able to identify probable counterparts consistent with the known Galactic and extragalactic X-ray source populations to all of the X-ray sources in the four SNRs discussed here. Therefore, there does not appear to be any detected neutron star in these SNRs. There are a small number of cases where either the association or the type of source (star versus galaxy) is uncertain, either due to an optical/IR detection in only one band and/or a detection at a somewhat large distance from the X-ray source, 


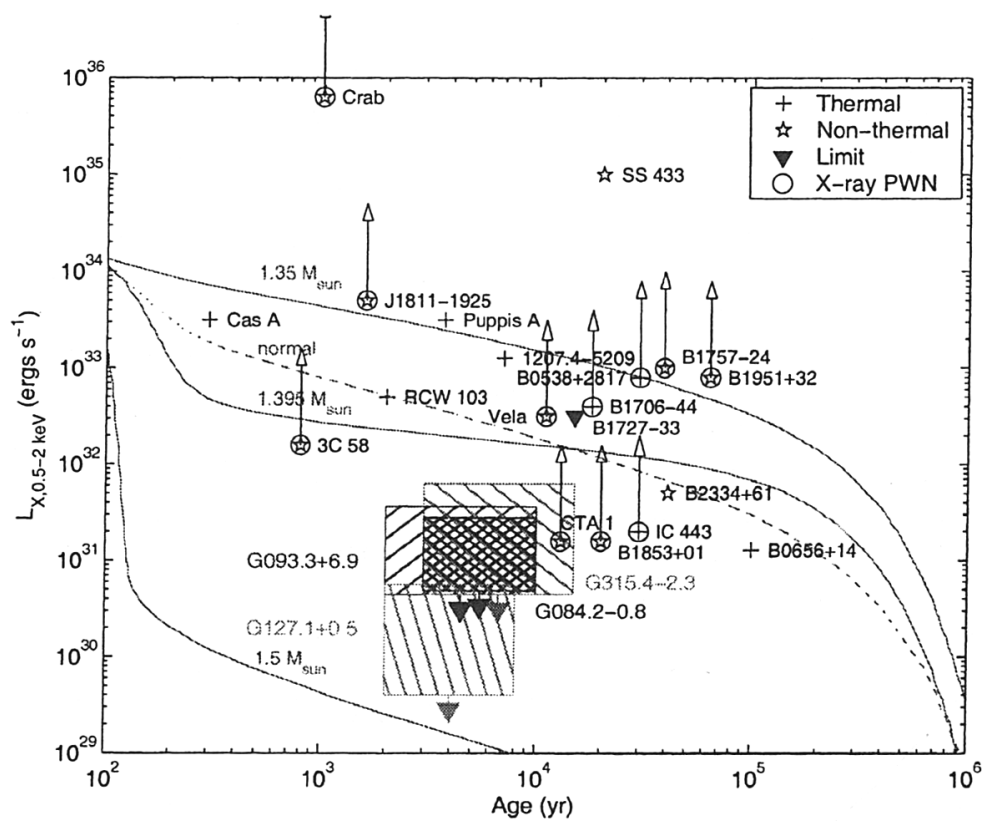

Figure 1. X-ray luminosities $(0.5-2 \mathrm{keV})$ as a function of age for neutron stars in SNRs from Kaplan et al. (2004). The sources that have $\mathrm{X}$-ray pulsar wind nebulae (PWNe), typically $>10$ times the X-ray luminosity of the neutron stars themselves, are circled and the PWN luminosities are indicated by arrows. We also plot the limits to blackbody emission from sources in SNRs G093.3+6.9 (dark-grey hatched region), G315.4-2.3 (upper light-grey hatched region), G084.2+0.8 (cross-hatched region), and G127.1+0.5 (lower light-grey hatched region). The cooling curves are the $1 \mathrm{p}$ proton superfluid models from Yakovlev et al. (2004; solid lines, with mass as labeled) and the normal (i.e., non-superfluid) $M=1.35 \mathrm{M}_{\odot}$ model (dot-dashed line), assuming blackbody spectra and $R_{\infty}=10 \mathrm{~km}$. They are illustrative of general cooling trends, and should not be interpreted as detailed predictions.

but there are certainly no sources that scream out "I am a neutron star." We then plot the resulting limits (incorporating uncertainties in distance, age, and source identification) in Figure 1 along with the luminosities of other neutron stars found in SNRs.

There are reasons why standard neutron stars were not found in these SNRs: they could have fallen in the gap between the chips, they could have escaped our field of view due to very high velocities ${ }^{1}$, they could be undetectable black

\footnotetext{
${ }^{1}$ To not be visible in SNRs G093.3+6.9, G315.4-2.3, or G084.2+0.8, the neutron star would have to be moving faster than $1500 \mathrm{~km} \mathrm{~s}^{-1}$, true for $\lesssim 1 \%$ of known pulsars. For SNR G127.1+0.5, the neutron star would have to be moving faster than $700 \mathrm{~km} \mathrm{~s}^{-1}$, true for $\lesssim 10 \%$ of known pulsars. See Arzoumanian, Cordes \& Chernoff (2002).
} 
holes, or they could not exist owing to the SNRs being the results of Type Ia explosions. All of these scenarios are unlikely for a single source, and even more so for all four, but are technically possible. If, on the other hand, these scenarios do not apply, then four remnants contain neutron stars that are fainter than our X-ray detection limit (typically, $L_{X} \lesssim 10^{31} \mathrm{ergs} \mathrm{s}^{-1}$ in the $0.5-10 \mathrm{keV}$ band).

We now consider this last (and most interesting) possibility. In the absence of other forms of energy generation (accretion, rotation power, magnetic field decay) the minimum X-ray flux one expects is set by the cooling of the neutron star. From Figure 1 we immediately see that the central neutron stars in these four remnants must be cooler than those present for example in the similarlyaged Puppis A, PKS 1209-51/52 and RCW 103.

Of course, we also do not see rotation-powered pulsars such as the majority of the objects in SNRs within $5 \mathrm{kpc}$. Recent observations are finding pulsars with lower radio luminosities and values of $\dot{E}$ than ever before, and our limits would only be consistent with these newer sources. One might then ask why we see neither a standard cooling neutron star nor a standard active pulsar, assuming that there is no intrinsic correlation between these properties. It is possible that there truly are no neutron stars in these SNRs, allowing one to speculate wildly about what actually is there.

Acknowledgments. D. L. K. is supported by a fellowship from the Fannie and John Hertz Foundation.

\section{References}

Arzoumanian, Z., Chernoff, D. F., \& Cordes, J. M. 2002, ApJ, 568, 289

Baade, W., \& Zwicky, F. 1934, PNAS, 20, 254

Barger, A. J., et al. 2003, AJ, 126, 632

Camilo, F., Kaspi, V. M., Lyne, A. G., Manchester, R. N., Bell, J. F., D'Amico, N., McKay, N. P. F., \& Crawford, F. 2000, ApJ, 541, 367

Gotthelf, E. V., Vasisht, G., Boylan-Kolchin, M., \& Torii, K. 2000, ApJ, 542, L37

Grindlay, J., et al. 2003, Astronomische Nachrichten, 324, 57

Hertz, P., \& Grindlay, J. E. 1988, AJ, 96, 233

Hulleman, F., van Kerkwijk, M. H., \& Kulkarni, S. R. 2000, Nature, 408, 689

Hurley, K. 2000, astro-ph/9912061

Kaplan, D. L., Frail, D. A., Gaensler, B. M., Gotthelf, E. V., Kulkarni, S. R., Slane, P. O., \& Nechita, A. 2004, ApJS, in press (astro-ph/0403313)

Mereghetti, S., Chiarlone, L., Israel, G. L., \& Stella, L. 2002, in MPE Rep. 278, Neutron Stars, Pulsars, and Supernova Remnants, eds. W. Becker, H. Lesch, \& J. Trümper, (Garching: MPE), p. 29

Pavlov, G. G., Sanwal, D., Garmire, G. P., \& Zavlin, V. E. 2002, in ASP Conf. Ser., Vol. 271, Neutron Stars in Supernova Remnants, eds. P. O. Slane, \& B. M. Gaensler, p. 247

Yakovlev, D. G., Gnedin, O. Y., Kaminker, A. D., Levenfish, K. P., \& Potekhin, A. Y. 2004, Adv. Sp. Res., 33, 523 